\title{
The Research of back propagation neural network based on genetic algorithm in the gas concentration prediction
}

\author{
Dapeng liu ${ }^{1, a}$, Fengying $\mathrm{ma}^{1,2, \mathrm{~b}}$ \\ ${ }^{1}$ School of Electrical Engineering and Automation, Qilu University of Technology, Jinan, China \\ ${ }^{2}$ Shandong University of Science and Technology, Qingdao 266590, China \\ a599139531@qq.com, b446240146@qq.com
}

Keywords: gas concentration, Genetic Algorithm, BP neural network, prediction model.

\begin{abstract}
The prediction of gas concentration is an extremely complicated nonlinear dynamic system which cannot fully use precise mathematical language to describe. Only using BP neural network algorithm is easy to converge to the local minimal point in the gas concentration prediction, so this paper presents the idea of GA-BP of neural network, which set up the GA - BP neural network model by optimizing the weights and threshold of BP neural network and its application in coal gas concentration prediction. This method combining genetic algorithm and neural network, and use genetic algorithm to optimize neural network to the initial value, so that the BP network can convergence to the optimal solution fast and can achieve higher precision in a shorter period, and the rate of convergence, accuracy and stability is superior to the BP network model, Thus verified the rationality and effectiveness of the GA - BP neural network.
\end{abstract}

\section{The introduction}

Due to its high nonlinear mapping, adaptive, self-learning habits, the BP netural network attracted much attention in recent years is frequently used in the gas concentration prediction. BP network has the shortcoming that it may converge to the local minimal point, and Genetic Algorithm can discover the optimal point of the overall situation,GA Can fully make up for the shortcomings of BP neural network. Therefore the combination of genetic algorithm and BP neural network, it can well realize the complementary advantages. So we build GA-BP model with GA-BP network, then we apply it to the prediction of gas concentration.

\section{The BP neural network}

BP neural network is a multilayer feedforward network according to the error backward Propagation training.The learning process of BP neural network is composed of two processes: forward propagation and error back propagation of error signals.In forward process, the data from the input layer propagate to the output layer through the hidden layer. The error propagate reversely and modify weights of each layer minimize error.Adjusting its weights constantly make the signal error toan acceptable level or at a preset number.

\section{Genetic algorithm}

The basic idea of genetic algorithms is forming search algorithm of "Generate and test" mode by a simulation for natural selection and genetic mechanisms. Genetic algorithms use code space to stand for the solution space of problem, and the code group is used as the basic object of evolution, and the fitness function is used as the basis of the evaluation, and the iterative process is established by genetic operation for the individual bit string of the group to achieve the genetic mechanism. In the iterative process, by the restructuring of the code in the series of important genes, a new string of bits set is better than the previous generation of bit string ensemble, gradually reach or approach the optimal solution. 


\section{Construction of neural GA_BP network}

Combining the genetic algorithm which is expert in overall global searching BP algorithm which is expert in local optimization can achieve two complementary advantages, improve the convergence speed, and faster to get the optimal solution. Optimizing the neural networks with genetic algorithm can constructed a neural network with not only the ability of learning, memory and nonlinear mapping, but also the ability of global searching. Utilizing genetic algorithm to optimizing BP neural network and the optimized result is called GA-BP neural network.

GA - BP neural network consists of the following three parts:

(1)Determine the structure of BP neural network: according to the problem to be solved, determine the length of the individual genetic algorithm.

(2)The BP neural network optimized by genetic algorithm: according to genetic algorithm, coding BP neural network's initial weights and threshold in order to determine the fitness function to calculate the individual fitness value finally finds the best individual through genetic operation.

(3)The BP neural network training: the optimal solution optimized by genetic algorithm, as optimal BP neural network weights and threshold, train the network, in order to achieve required accuracy.

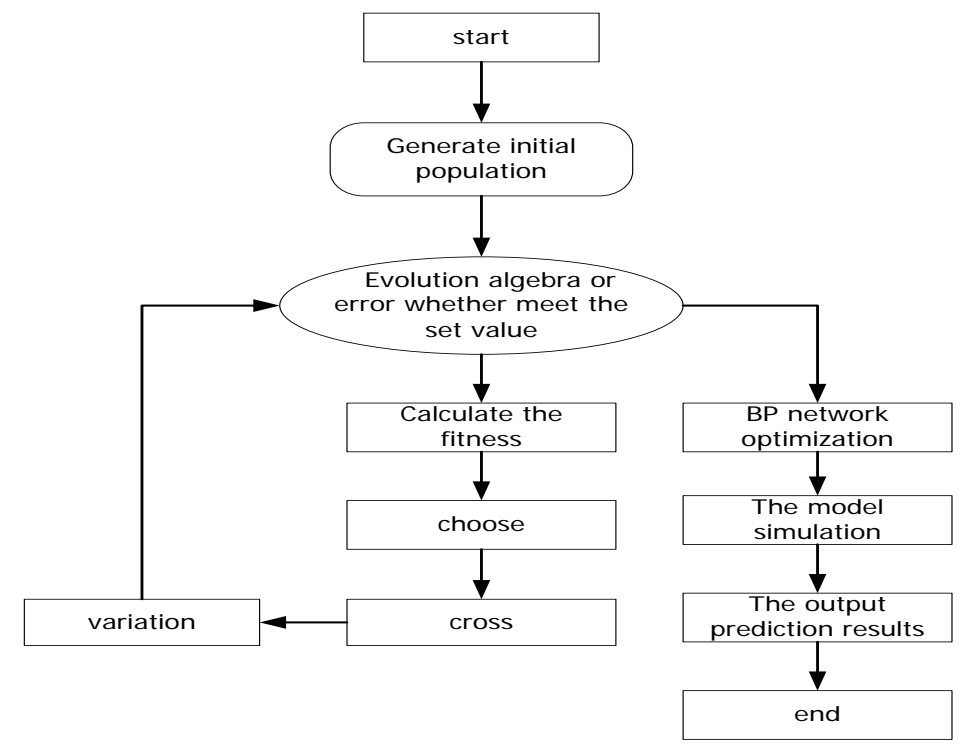

Figure 1: The process of genetic algorithm optimizing the network weight

The number of network layer and hidden layer nodes. Existing research results show that three-layer feedforward neural network can approach any nonlinear relation with any degree of accuracy, so the prediction model of this paper is a three-layer feedforward neural network structure.The input layer is responsible for receiving information directly affecting the gas concentration, the output layer yields a final result needed, there is a layer between input and output layer, they are called hidden layer generally.

There are five major factors that affect gas concentration in the coal mine production: the depth of coal seam, the thickness of coal seam, coal seam gas concent, daily output and wind speed. So the number of input node is five. The number of Output layer is 1 , which corresponds to the gas concentration. There is no mature theory and method to determine the number of hidden layer. We usually use empirical formula $q=\sqrt{m+n}+\alpha$ to solve this problem. $\mathrm{N}$ is the number of input layer node, $\mathrm{m}$ is the number of output layer node, $\alpha$ is a constant between 1-10,so the range of the number of hidden layer nodes is $[4,13]$.After a lot of testing, we found that when the number of hidden layer nodes taken 9, network stability and the accuracy were higher, therefore, the number of hidden layer nodes is 9.The topologica1 structure of the model is $(5,9,1)$. The network structure as shown in figure 2 


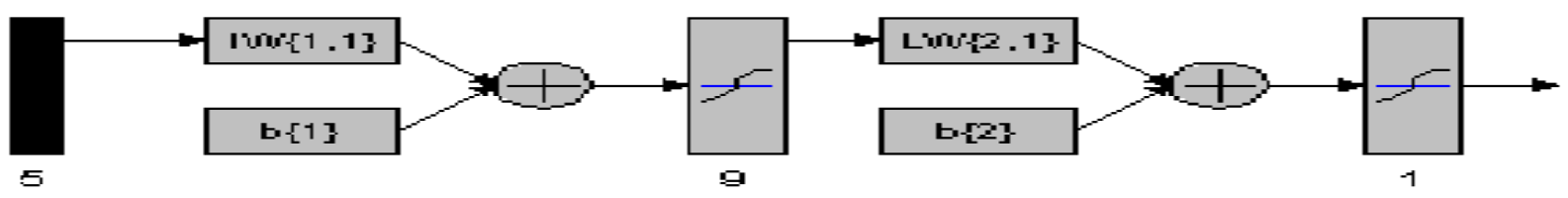

Figure 2 the network structure

Data preprocessing. The original data samples of each physical quantity have different orders of magnitude, and the difference is extremely tremendous. To avoid input data into saturation area, at the same time to keep the original characteristics of the data, we should make a data preprocessing in order to improve the training speed of network and to facilitate the calculation of the network. After normalization processing of the original data input and output by premnmx function, the data processed are evenly distributed in the range of $[1,1]$. The conversion formula is $: \bar{x}=\frac{x-\min (x)}{\max (x)-\min (x)}, \max (\mathrm{x})$ and $\min (\mathrm{x})$ is the maximum and the minimum of sample data respectively, $\mathrm{x}$ is the original sample data, $\bar{x}$ is the value transformed. After the BP neural network training, the simulation is still the normalized results. Meanwhile the results need to be for the reverse normalization processing by postmnmx function to make it back to normal.

Coding and the generation of initial population. Network connection rights needs to be encoded, so floating-point coding is chosen to encode connection power.The BP neural network weights and threshold of cascade in a certain order to form a floating point count group as a chromosome of genetic algorithm.GA - BP model uses the three layers BP network structure, set the number of input layer, hidden layer and output layer are $\mathrm{i}, \mathrm{j}$ and $\boldsymbol{m}_{k}$, the length of coding is ${ }^{m=m_{k} \times i+m_{k} \times j+j} \mathrm{X}$ chromosome which length is $\mathrm{R}$ is randomly generated (forming the initial population). Because the topological structure of the model is $(5,9,1)$, the coding length is $m=9 \times 5+9 \times 1+1=51$. Population number $Y$ must have an appropriate value, too much value will lead to network slow convergence speed, small value will reduce the accuracy of network training.So $\mathrm{Y}$ is 20.

\section{The determination of fitness function}

The Prediction model is trained by choosing the gas concentration of training samples. Then type testing data to verify the GA - BP prediction effect.

Table 1: Training data

\begin{tabular}{|c|c|c|c|c|c|c|}
\hline No. & $\mathrm{h} / \mathrm{m}$ & $\mathrm{d} / \mathrm{m}$ & $\mathrm{q} /\left(t \cdot d^{-1}\right)$ & $\mathrm{V} /\left(m^{3} \cdot t^{-1}\right)$ & $\mathrm{v} /\left(m \cdot t^{-1}\right)$ & $\varphi / \%$ \\
\hline 1 & 110 & 1.38 & 237 & 22.7 & 1.8 & 0.346 \\
\hline 2 & 105 & 1.16 & 206 & 21.7 & 1.2 & 0.373 \\
\hline 3 & 124 & 1.03 & 175 & 19.8 & 2.2 & 0.389 \\
\hline 4 & 116 & 0.93 & 182 & 18.8 & 2.3 & 0.431 \\
\hline$\ldots$ & $\ldots$ & $\ldots$ & $\ldots$ & $\ldots$ & $\ldots$ & $\ldots$ \\
\hline 20 & 121 & 1.11 & 143 & 22.4 & 1.9 & 0.471 \\
\hline
\end{tabular}

On the basis of trained network, gas concentration prediction results are obtained as shown in table 2.Therefore, GA - BP predicted values are closer to the actual value!

Table 2 the comparison of prediction model error

\begin{tabular}{|c|c|c|c|c|c|}
\hline No. & $\begin{array}{c}\text { measured } \\
\text { values }\end{array}$ & BP & relative error\% & GA_BP & relative error\% \\
\hline 1 & 0.310 & 0.328 & 5.8 & 0.322 & 3.8 \\
\hline 2 & 0.317 & 0.341 & 7.6 & 0.315 & 0.6 \\
\hline 3 & 0.519 & 0.446 & 14 & 0.514 & 0.9 \\
\hline 4 & 0.690 & 0.666 & 3.4 & 0.697 & 1.0 \\
\hline
\end{tabular}




\section{Conclusion}

Using the genetic algorithm to optimize neural network to the initial value can achieve higher precision in a shorter period and convergence the BP network to the optimal solution fast, and the rate of convergence, accuracy and stability are superior to the BP network model, thus verified the rationality and effectiveness of the GA - BP neural network.Applying this method ideal results of gas concentration prediction has been achieved.Compared with the BP neural network, GA - BP prediction error is smaller to improve the accuracy of prediction to guarantee the safe production.

\section{References}

[1] leijiang.Coal mine gas concentration prediction model building and simulation based on the BP neural network[J].the mining safety and environmental protection,2010,37 (4):37 -39.

[2] Binghan, Huafu.The gas monitoring system based on BP neural network data fusion[J].Industrial automation,2008 (4) : 10-13.

[3] Yili, Yuxiaoliu.Gas emission prediction research based on BP neural network data fusion[J]. Modernization of coal mine,2011(2):23-24

[4] Zhanglicai, Xiaolinchen, Weirenshi.Learning effect comprehensive evaluation method of improvement based on BP neural network [J].Journal of chongqing university(Natural science edition),2007.30(7):96-99.

[5] minqiang li, the basic theory and application of genetic algorithms [M]. Beijing: science press, 2002 